\title{
CONSISTENT RELATIVISTIC VLBI THEORY WITH PICOSECOND ACCURACY $\dagger$
}

\author{
M. SOFFEL ${ }^{1}$, J. MÜLLER ${ }^{2}, X . W^{1}$, C. XU ${ }^{1}$ \\ ${ }^{1}$ Theoretical Astrophysics \\ Uni. Tübingen \\ Auf der Morgenstelle 10 \\ 7400 Tübingen, FRG \\ ${ }^{2}$ Technical Uni. Munich \\ Institut f. Astron. u. Physikal. Geodäsie \\ Arcisstr. 21 \\ 8000 Munich 2, FRG
}

\section{Introduction}

The accuracy of Very Long Baseline Interferometry (VLBI), representing one of the most important space techniques of modern geodesy, especially for the determination of the Earth's rotation parameters and baselines, is steadily increasing. Presently, delay residuals are of the order of $30-50 \mathrm{ps}$, corresponding to an uncertainty in length of about 1 centimeter e.g. in the determination of baselines or the position of the rotation pole. As has already been stressed by many authors, at this level of accuracy a relativistic formulation of the VLBI measuring process is indispensable (e.g. the gravitational time delay for rays getting close to the limb of the Sun amounts to $170 \mathrm{~ns}$ !). Starting with the work by Finkelstein et al. (1983) a series of papers has meanwhile been published on a relativistic VLBI theory (Soffel et al., 1986; Hellings, 1986; Zeller et al., 1986; Herring, 1989). However, possibly apart from Brumberg's treatment in his new monograph (Brumberg, 1990) all of these theories have one fatal drawback: they are not based upon some consistent theory of reference frames, which relates the global, barycentric coordinates, in which the measuring process is primarily formulated and in which positions and velocities of the bodies of the solar system are computed, with the local, geocentric coordinates, comoving with

† Paper presented at the Eubanks Meeting at the U.S. Naval Observatory, Washington, D.C., October 12th, 1990. Work supported by the Deutsche Forschungsgemeinschaft (DFG) and the Volkswagen Stiftung; M.S. kindly acknowledges the receipt of a Heisenberg fellowship 
the Earth, in which the geodetically meaningful baselines are defined. Furthermore, none of these theories (including Brumberg's (1990) treatment) have the accuracy of one picosec which seems desirable with respect to the achieved residual values.

This article presents a relativistic VLBI theory with an accuracy of better than 1 ps. It is based upon a consistent theory of reference frames in the solar system which first has been introduced by Damour, Soffel and Xu (1990a-c) and complete at the first post-Newtonian level. For an accuracy of 1 ps our result differs from all results that have been published earlier.

\section{Post-Newtonian VLBI Theory For The Group Delay}

We consider some radio signal being emitted from some remote source at barycentric coordinate time $t_{0}$ and position $\boldsymbol{x}_{0}$. We consider two "light-rays", contained in the signal, which arrive at two VLBI antennas (called 1 and 2) at coordinate time $t_{1}$ and $t_{2}$. This barycentric coordinate time $t$ is also called TCB. Let us denote the Euclidean unit vector from the source to the barycenter by $k\left(k^{i} k^{i}=1\right)$. Then the barycentric coordinate arrival time difference $t_{2}-t_{1}$ to first post-Newtonian order is given by

$$
\Delta t \equiv t_{2}-t_{1}=-\frac{1}{c}\left[x_{1}\left(t_{1}\right)-x_{2}\left(t_{2}\right)\right] \cdot k+(\Delta t)_{\mathrm{grav}},
$$

where $x_{i}\left(t_{i}\right)$ denotes the barycentric coordinate position of antenna $i$ at coordinate time $t_{i} .(\Delta t)_{\mathrm{grav}}$ is the gravitational time delay, resulting from solving the equation for null geodesics (light rays) in some background metric describing the gravitational influence of the Sun and the planets. To sufficient accuracy $(\Delta t)_{\mathrm{grav}}$ can be written as a sum over the contributions of the various massive bodies in the solar system.

Now, for picosecond accuracy it is sufficient to consider the spherical part of the gravitational potential in $(\Delta t)_{\mathrm{grav}}$ only. Taking earlier results from Richter and Matzner (1983) we estimate the contribution from the quadrupole moment of the Sun to the time delay to be much less than a picosecond $\left(\sim 10^{-18} \mathrm{~s}\right)$. The effect from the angular momentum of the Sun (a gravitomagnetic effect of 1.5 post-Newtonian order) is of the same order, while the dominant post-post Newtonian terms are expected to be less than about 0.5 picoseconds.

For the solar contribution we can neglect the motion of the Sun about the barycenter and the usual "light-time equation" for the spherical field can be written in the form (e.g. Soffel, 1989)

$$
(\Delta t)_{\mathrm{grav}}^{\odot} \simeq \frac{2 m_{\odot}}{c} \ln \left(\frac{\left|x_{1}\right|-x_{1} \cdot k}{\left|x_{2}\right|-x_{2} \cdot k}\right)
$$

where $\boldsymbol{x}_{i}$ refers to $t_{i}$ and $m_{\odot} \equiv G M_{\odot} / c^{2}=1.48 \mathrm{~km}$. The time difference $\Delta t$ can be neglected in the ln-term and writing

$$
\boldsymbol{x}_{i}=\boldsymbol{x}_{\oplus}+\Delta \boldsymbol{r}_{i}
$$


we obtain (Finkelstein et al., 1983; Zeller et al., 1986):

$$
\begin{aligned}
& (\Delta t)_{\mathrm{grav}}^{\odot} \\
& \simeq \frac{2 m_{\odot}}{c} \ln \left[\frac{r_{\oplus}\left(1-e_{\oplus} \cdot k\right)+\Delta r_{1} \cdot\left(e_{\oplus}-k\right)+\left(\Delta r_{1}\right)^{2} / 2 r_{\oplus}-\left(e_{\oplus} \cdot \Delta r_{1}\right)^{2} / 2 r_{\oplus}}{r_{\oplus}\left(1-e_{\oplus} \cdot k\right)+\Delta r_{2} \cdot\left(e_{\oplus}-k\right)+\left(\Delta r_{2}\right)^{2} / 2 r_{\oplus}-\left(e_{\oplus} \cdot \Delta r_{2}\right)^{2} / 2 r_{\oplus}}\right]
\end{aligned}
$$

with

$$
\boldsymbol{e}_{\oplus} \equiv \boldsymbol{x}_{\oplus} / r_{\oplus} ; \quad r_{\oplus}=\left|x_{\oplus}\right|=\left(x_{\oplus}^{i} x_{\oplus}^{i}\right)^{1 / 2}
$$

For baselines of $\sim 6000 \mathrm{~km}$, the $\left(\Delta \boldsymbol{r}_{i}\right)^{2}$-terms are of order $3 \times 10^{-14} \mathrm{sec}$ and can be neglected for picosec-accuracy. $0.44 \mathrm{~cm}$ )

For the gravitational time delay due to the Earth one finds $\left(m_{\oplus}=G M_{\oplus} / c^{2}=\right.$

$$
(\Delta t)_{\mathrm{grav}}^{\oplus} \simeq \frac{2 m_{\oplus}}{c} \ln \left[\frac{\left|\Delta r_{1}\right|-\Delta r_{1} \cdot k}{\left|\Delta r_{2}\right|-\Delta r_{2} \cdot k}\right],
$$

if the motion of the Earth during signal propagation is neglected. Similarly, for any other planet $A$, if its motion is neglected, one obtains

$$
(\Delta t)_{\mathrm{grav}}^{A} \simeq \frac{2 m_{A}}{c} \ln \left[\frac{\left|x_{A 1}\right|-x_{A 1} \cdot k}{\left|x_{A 2}\right|-x_{A 2} \cdot k}\right]
$$

where $x_{A i}=x_{i}-x_{A}$. Note that the maximal gravitational time delays due to Jupiter, Saturn, Uranus and Neptune are of order 1.6(Jup), .6(Sat), .2(U) and .2(N) nanosec resprectively, but these values decrease rapidly with increasing angular distance from the limb of the planet. E.g. 10 arcmin from the center of the planet the gravitational time delay amounts only to about 60 picosec for Jupiter, 9 picosec for Saturn and about one picosec for Uranus. To consider the barycentric motion of the planet during signal propagation the position of the planet might be taken at the time of closest approach (e.g. Hellings, 1986); it is, however, unclear how good this correction for the planet's velocity really is.

Let us define baselines at signal arriving time $t_{1}$ at antenna 1 . Let the barycentric baseline $b$ be defined as

$$
\boldsymbol{b}\left(t_{1}\right) \equiv \boldsymbol{x}_{1}\left(t_{1}\right)-\boldsymbol{x}_{2}\left(t_{1}\right)
$$

then a Taylor expansion of $x_{2}\left(t_{2}\right)$ about $t_{1}$ yields $\left(O(n) \equiv O\left(c^{-n}\right)\right)$

$$
\begin{aligned}
\Delta t= & -\frac{1}{c}(b \cdot k)\left[1+\frac{1}{c}\left(\dot{x}_{2} \cdot k\right)+\frac{1}{c^{2}}\left(\dot{x}_{2} \cdot k\right)^{2}-\frac{1}{2 c^{2}}(b \cdot k)\left(\ddot{x}_{2} \cdot k\right)\right] \\
& +(\Delta t)_{\mathrm{grav}}+O(4)
\end{aligned}
$$


all quantities now referring to barycentric coordinate time $t_{1}$. We call this relation the "VLBI-delay equation", describing the barycentric coordinate time delay $\Delta t$ entirely by quantities defined in the global system.

We will now relate the various barycentric quantities with corresponding geocentric ones apart from the propagation vector $k$. This will remind us that the process of signal propagation from the source to the antennas cannot be formulated in the local, accelerated, geocentric system. We now write the time transformation in the form (Damour et al., 1990a-c)

$$
\begin{aligned}
c t & =z_{\oplus}^{0}(T)+e_{a}^{0}(T) X^{a}+O(3) \\
& =\int_{T_{0}}^{T} c e_{0}^{0} d T^{\prime}+e_{a}^{0}(T) X^{a}+O(3) \\
& =c\left(T-T_{0}\right)+\frac{1}{c} \int_{T_{0}}^{T}\left(\bar{U}\left(z_{\oplus}\right)+\frac{1}{2} v_{\oplus}^{2}\right) d T^{\prime}+\frac{1}{c} R_{a}^{i}(T) v_{\oplus}^{i}(T) X^{a}+O(3)
\end{aligned}
$$

Here, $T=$ TCG is the geocentric coordinate time and $X^{a}$ the geocentric spatial coordinate. $\bar{U}$ is the external gravitational potential which does not include the contribution from the Earth. Replacing $T^{\prime}$ by $t^{\prime}$ in the integral and considering that $R_{a}^{i}$ is a slowly time dependent matrix we can relate $\Delta t=t_{2}-t_{1}$ with the corresponding local time interval $\Delta T=\Delta \mathrm{TCG}=T_{2}-T_{1}$ :

$$
\begin{aligned}
\Delta t= & \Delta T+\frac{1}{c^{2}} \int_{t_{1}}^{t_{2}}\left(\bar{U}\left(\boldsymbol{z}_{\oplus}\right)+\frac{1}{2} \boldsymbol{v}_{\oplus}^{2}\right) d t^{\prime} \\
& +\frac{1}{c^{2}} R_{a}^{i} v_{\oplus}^{i}\left(T_{2}\right) X_{2}^{a}\left(T_{2}\right)-\frac{1}{c^{2}} R_{a}^{i} v_{\oplus}^{i}\left(T_{1}\right) X_{1}^{a}\left(T_{1}\right)+O(4)
\end{aligned}
$$

With

$$
v_{\oplus}^{i}\left(T_{2}\right) X_{2}^{a}\left(T_{2}\right) \simeq v_{\oplus}^{i} X_{2}^{a}-v_{\oplus}^{i} V_{2}^{a}\left(\frac{b \cdot k}{c}\right)-a_{\oplus}^{i} X_{2}^{a}\left(\frac{b \cdot k}{c}\right)+O(2)
$$

where quantities on the right hand side now refer to $T_{1}$, we formally get the relation

$$
\begin{aligned}
\Delta t= & \Delta T+\frac{1}{c^{2}}\left[-\boldsymbol{v}_{\oplus} \cdot \boldsymbol{B}+\int_{t_{1}}^{t_{2}}\left(\bar{U}\left(\boldsymbol{z}_{\oplus}\right)+\frac{1}{2} \boldsymbol{v}_{\oplus)}^{2}\right) d t^{\prime}\right] \\
& -\frac{1}{c^{3}}\left[\left(\boldsymbol{v}_{\oplus} \cdot \boldsymbol{v}_{2}\right)(\boldsymbol{b} \cdot \boldsymbol{k})+\left(\boldsymbol{a}_{\oplus} \cdot \Delta \boldsymbol{r}_{2}\right)(\boldsymbol{b} \cdot \boldsymbol{k})\right]+O(4),
\end{aligned}
$$

where

$$
\boldsymbol{B}\left(T_{1}\right) \equiv \boldsymbol{X}_{1}\left(T_{1}\right)-\boldsymbol{X}_{2}\left(T_{1}\right)
$$


and

$$
\begin{aligned}
B^{i} & \equiv R_{a}^{i} B^{a} \\
v_{2}^{i} & \equiv R_{a}^{i} V_{2}^{a} \\
\Delta r_{2}^{i} & \equiv R_{a}^{i} X_{2}^{a} .
\end{aligned}
$$

$v_{2}$ is the geocentric velocity of antenna 2. Next, we will relate the barycentric baseline vector $b\left(t_{1}\right)$ appearing in the VLBI-delay equation (7), with the corresponding geocentric one $B\left(T_{1}\right)$. Using the notation of Damour et al. (1990a-c) we find

$$
\begin{aligned}
x_{1}^{i}\left(t_{1}\right)-x_{2}^{i}\left(t_{2}\right)= & z_{\oplus}^{i}\left(t_{1}\right)-z_{\oplus}^{i}\left(t_{2}\right)+R_{a}^{i}\left(X_{1}^{a}\left(T_{1}\right)-X_{2}^{a}\left(T_{2}\right)\right) \\
& -\frac{1}{c^{2}}\left(\frac{1}{2} v_{\oplus}^{i} v_{\oplus}^{k}+\bar{U}\left(z_{\oplus}\right) \delta_{i k}\right) B^{k}+\xi^{i}\left(T_{1}, X_{1}^{a}\right)-\xi^{i}\left(T_{2}, X_{2}^{a}\right)
\end{aligned}
$$

with

$$
\xi^{i}=\frac{1}{c^{2}} e_{a}^{i}(T)\left[\frac{1}{2} A_{\oplus}^{a} X^{2}-X^{a}\left(A_{\oplus} \cdot X\right)\right]+O(4)
$$

and

$$
A_{\oplus}^{a}(T) \equiv e_{a}^{i}(T) \frac{d^{2} z_{\oplus}^{i}}{d T^{2}}
$$

With

$$
\begin{aligned}
z_{\oplus}^{i}\left(t_{1}\right)-z_{\oplus}^{i}\left(t_{2}\right) & \simeq-v_{\oplus}^{i} \Delta t-\frac{1}{2} a_{\oplus}^{i}(\Delta t)^{2} \\
x_{1}^{i}\left(t_{1}\right)-x_{2}^{i}\left(t_{2}\right) & \simeq b^{i}\left(t_{1}\right)-(\Delta t) \dot{x}_{2}^{i}\left(t_{1}\right)-\frac{1}{2}(\Delta t)^{2} \ddot{x}_{2}^{i}\left(t_{1}\right)
\end{aligned}
$$

and

$$
X_{1}^{a}\left(T_{1}\right)-X_{2}^{a}\left(T_{2}\right) \simeq B^{a}\left(T_{1}\right)-\left(\frac{d}{d T} X_{2}^{a}\right) \Delta T-\frac{1}{2}\left(\frac{d^{2}}{d T^{2}} X_{2}^{a}\right)(\Delta T)^{2}
$$

where

$$
\Delta T=\Delta t+\frac{1}{c^{2}} \boldsymbol{v}_{\oplus} \cdot \boldsymbol{B}+O(3)
$$

(the integral in (8) is practically of order $c^{-3}$ ) the desired relation between barycentric and geocentric baselines reads

$$
b^{i}=B^{i}-\frac{1}{c^{2}}\left(v_{\oplus} \cdot B\right) v_{2}^{i}-\frac{1}{c^{2}}\left(\frac{1}{2} v_{\oplus}^{i} v_{\oplus}^{k}+\bar{U}\left(z_{\oplus}\right) \delta_{i k}\right) B^{k}+\Delta \xi^{i}+O(3)
$$

with

$$
\Delta \xi^{i} \equiv \xi^{i}\left(T_{1}, X_{1}^{a}\right)-\xi^{i}\left(T_{2}, X_{2}^{a}\right)
$$


Using equation (8), the VLBI-delay equation (7) for $(\Delta t)$ and the relation (11) for the baselines we obtain the formal expression $\left(B \cdot k=B^{i} k^{i}\right.$ etc.):

$$
\begin{aligned}
& \Delta T= \\
& -\frac{1}{c}(B \cdot k)-\frac{1}{c^{2}}(B \cdot k) k \cdot\left(v_{\oplus}+v_{2}\right)+\frac{1}{c^{2}}\left(v_{\oplus} \cdot B\right) \\
& +\frac{1}{c^{3}}\left(v_{\oplus} \cdot v_{2}\right)(B \cdot k)-\frac{1}{c^{3}}(B \cdot k)\left[k \cdot\left(v_{\oplus}+v_{2}\right)\right]^{2}+\frac{1}{c^{3}}(B \cdot k) \bar{U}\left(z_{\oplus}\right) \\
& +\frac{1}{2 c^{3}}\left(v_{\oplus} \cdot k\right)\left(v_{\oplus} \cdot B\right)+\frac{1}{c^{3}}\left(v_{\oplus} \cdot B\right)\left(v_{2} \cdot k\right)-\frac{1}{c^{2}} \int_{t_{1}}^{t_{2}}\left(\bar{U}\left(z_{\oplus}\right)+\frac{1}{2} v_{\oplus}^{2}\right) d t^{\prime} \\
& -\frac{1}{c} k^{i} \Delta \xi^{i}+\frac{1}{2 c^{3}}(B \cdot k)^{2} k\left(a_{\oplus}+\dot{v}_{2}\right)+\frac{1}{c^{3}}(B \cdot k)\left(a_{\oplus} \cdot \Delta r_{2}\right) \\
& +(\Delta t)_{\operatorname{grav}}+O(4) .
\end{aligned}
$$

Keeping only terms with amplitudes greater than 1 picosec for baselines of the order of $6000 \mathrm{~km}$, we approximately find:

$$
\begin{aligned}
& \Delta T= \\
& -\frac{1}{c}(B \cdot k)-\frac{1}{c^{2}}(B \cdot k) k \cdot\left(v_{\oplus}+v_{2}\right)+\frac{1}{c^{2}}\left(v_{\oplus} \cdot B\right) \\
& +\frac{1}{c^{3}}\left(v_{\oplus} \cdot v_{2}\right)(B \cdot k)-\frac{1}{c^{3}}(B \cdot k)\left[k \cdot\left(v_{\oplus}+v_{2}\right)\right]^{2}+\frac{1}{c^{3}}(B \cdot k)\left(2 \bar{U}\left(z_{\oplus}\right)+\frac{1}{2} v_{\oplus}^{2}\right) \\
& +\frac{1}{2 c^{3}}\left(v_{\oplus} \cdot k\right)\left(v_{\oplus} \cdot B\right)+\frac{1}{c^{3}}\left(v_{\oplus} \cdot B\right)\left(v_{2} \cdot k\right)+(\Delta t)_{\mathrm{grav}}
\end{aligned}
$$

Finally, the geocentric coordinate time $T$ can be related with proper time $\tau$ as indicated by some (atomic) clock located at some VLBI station. Neglecting all tidal effects on local clock rates for clocks at rest at the Earth's surface we find

$$
\frac{d \tau}{d T} \simeq 1-\frac{1}{c^{2}}\left(U_{\oplus}(X)+\frac{1}{2}(\Omega \times X)^{2}\right)=1-\frac{1}{c^{2}} U_{\text {geo }}(X),
$$

where $U_{\oplus}$ is the gravitational potential of the Earth and $\boldsymbol{\Omega}$ is the angular velocity of the Earth's rotation. This can be written in the form

$$
d \tau \simeq\left[1-\frac{1}{c^{2}} U_{\mathrm{geo}}^{0}+\frac{1}{c^{2}} g(\psi) h\right] d T,
$$

where $U_{\text {geo }}^{0}$ is the geopotential at the geoid, $g(\psi)=\left(9.78027+0.05192 \sin ^{2} \psi\right) \times$ $10^{2} \mathrm{~cm} / \mathrm{s}^{2}$ is the latitude dependent gravity acceleration and $h$ is the height above 
the geoid. Instead of using this formula for the $T \leftrightarrow \tau$ relation, we split it into two parts, defining $\tau^{*}=\mathrm{TT}$ as proper time on the geoid:

$$
\begin{aligned}
d \tau^{*}=d(\mathrm{TT}) & \equiv\left(1-\frac{1}{c^{2}} U_{\mathrm{geo}}^{0}\right) d T \equiv \kappa_{0} d T=\kappa_{0} d(\mathrm{TCG}) \\
d \tau & =\left(1+\frac{1}{c^{2}} g(\psi) h\right) d \tau^{*} .
\end{aligned}
$$

The constant $\kappa_{0}$ relating $\tau^{*}$ with the geocentric coordinate time $T$ has the numerical value

$$
\kappa_{0} \simeq 1-6.9 \times 10^{-10} .
$$

Finally we would like to adress the question of the orientation of spatial coordinates of the local geocentric system. This orientation is determined by the matrix $R_{a}^{i}$ (remember that in eqs.(12) and (13) $\boldsymbol{B} \cdot \boldsymbol{k}=B^{i} k^{i}$ with $B^{i}=R_{a}^{i} B^{a}$ ). There are two preferred choices for $R_{a}^{i}$ leading to geocentric coordinates which are either

- fixed star oriented (kinematically non-rotating)

- or locally inertial (dynamically non-rotating).

In the first case of kinematically non-rotating coordinates we can take $R_{a}^{i}=\delta_{a}^{i}$. Then the geodesic precession will be in the precession-nutation matrices as well as in the dynamical equations (e.g. for satellies orbiting the Earth); it will not appear in the group delay equations (12) or (13). On the other hand if dynamically non-rotating geocentric coordinates are chosen then the geodesic precession (secular and annual term) has to be included in the $R_{a}^{i}$ matrix. In this case the precession-nutation matrices (and dynamical equations) do not contain the geodesic precession.

\section{References}

Brumberg, V.A., 1990, "Essential Relativistic Celestial Mechanics", Hilger, Bristol Brumberg, V.A., Kopejkin, S.M., 1988, in: "Reference Systems", J.Kovalevsky, I.I. Mueller and B.Kolaczek (eds.), Reidel, Dordrecht

Brumberg, V.A., Kopejkin, S.M., 1989, Nuovo Cim. B103, 63

Blanchet,L., Damour,T., 1989, Ann. Inst. Henri Poincaré, 50, 377

Damour,T., Soffel,M., Xu,C., 1990a, in: "Les Journées 1990", Systemes de Reference Spatio-Temporels, N.Capitaine (ed.), Observatoire de Paris 
Damour,T., Soffel,M., Xu,C., 1990b, "General Relativistic Celestial Mechanics I. Method and Definition of Reference Systems", submitted for publication

Damour,T., Soffel,M., Xu,C., 1990c, "Relativistic Celestial Mechanics and Reference Frames", Proc. of the IAU Colloqium 127, Virginia Beach, Oct., 14-20, 1990

Finkelstein,A.M., Kreinovich,V.J., Pandey,S.N., 1983, Astrophys. Space Sci. 94, 233

Hellings, R.W., 1986, Astron. J. 91, 650

Herring, T., 1989, Memo to Jim Ryan, dated January, 30, 1989 based upon previous notes by I.I.Shapiro

Misner,C., Thorne,K.S., Wheeler,J.A., 1973, "Gravitation", Freeman, San Francisco

Richter, G.W., Matzner, R.A., 1983, Phys. Rev. 28, 3007

Soffel,M., Ruder,H., Schneider,M., Campbell,J., Schuh,H., 1986, in: "Relativistic effects in Celestial Mechanics and Geodesy", J.Kovalevsky and V.Brumberg (eds.), Reidel, Dordrecht

Soffel,M., 1989, "Relativity in Astrometry, Celestial Mechanics and Geodesy", Springer, Heidelberg, Berlin, New York

Will,C., 1981, "Theory and Experiment in Gravitational Physics", Cambridge University Press, Cambridge

Zeller,G., Soffel,M., Ruder,H., Schneider,M., 1986, Veröff. der Bayr. Komm. f.d. Intern. Erdmessung, Astronomisch-Geodätische Arbeiten, Heft Nr.48, pp. 218-236 